\title{
CURRENT TRENDS IN DIETARY MANAGEMENT OF DIABETES MELLITUS AND ITS COMPLICATIONS
}

\section{*P. Stephen}

${ }^{*}$ Consultant Nutritionist-Dietician, Department of Dietetics, University of Benin Teaching Hospital, Benin City, Nigeria

\section{Correspondence:}

Mr Peter Stephen

Department of Dietetics,

University of Benin Teaching Hospital,

Benin City, Nigeria

Email: petliz2003@yahoo.com

\section{INTRODUCTION}

Medical nutrition therapy is integral to total diabetes care and management. Diet is the one of the treatment common to all diabetics and in the mildest cases is all that is needed to restore carbohydrate tolerance to normal. Dietary control plays a role in any of the three ways currently used in the management of diabetes which are as follows:

- Diet alone

- Diet and oral hypoglycemic drugs

- Diet and insulin

It is important to note that the dietary recommendations for persons with diabetes are in most respect the same as for non-diabetic persons and are based on sound principles of nutrition. Although adherence to nutrition and meal planning principles is one of the most challenging aspects of diabetes care, nutrition therapy is an essential component of successful diabetes management.

\section{AIMS OF DIETARY TREATMENT}

In managing diabetes mellitus, the aim of dietary management is to:

(1) Improve the overall health by achieving and maintaining optimal nutritional status.
(2) Achieve and maintain ideal level of body fat or ideal body weight

(3) Maintain blood glucose level as near normal range as possible

(4) Minimize glycosuria

(5) Prevent, delay and treat the acute complications of insulin treated diabetes such as hypoglycemia and long term complications such as renal diseases, retinal, hypertension and cardiovascular diseases.

To achieve these goals in the insulin dependent diabetic, it is important to consider the timing of meals, the composition of the diet, the energy content of the diet and the physical activity.

\section{IMPORTANT CONSIDERATIONS AND NUTRITION RECOMMENDATION AND PRINCIPLES FOR PEOPLE WITH DIABETES MELLITUS}

Caloric allowances: The dietary recommendation for persons with diabetes is in most respect the same as for non-diabetic persons and is based on sound principles of nutrition. The same procedure used to plan the caloric allowance for a normal diet is followed when computing a diet prescription for a diabetic patient and is first determined based on the 
patient's height, weight, age, sex, occupation or activity level.

Protein allowances: The protein determination for a diabetic is essentially the same as that for the normal individual and may vary from 0.8 to $1.7 \mathrm{~g} / \mathrm{kg}$ of the desired body weight or 0.4 to $0.7 \mathrm{gm} / \mathrm{lb}$ depending on age. The consensus guideline recommends that protein intake should provide $10-20 \%$ of the total daily energy intake derived from both animal and vegetable sources. With the onset of nephrology, a lower intake of about $0.8 \% / \mathrm{kg} /$ day should be considered. This is approximately $10 \%$ of total energy intake.

Carbohydrate: An amount less than $100 \mathrm{gm}$ is inadvisable since a low level frequently leads to the ketosis and an amount over $300 \mathrm{gm}$ may overtax the metabolic capacity.

The diet should provide $50-60 \%$ of the total daily intake as carbohydrate from a variety of sources. The daily consumption of a diet high in complex carbohydrate and ideally containing approximately $3 \mathrm{~g}$ dietary fiber (non starch polysaccharide) per $1000 \mathrm{~kJ}$ is recommended. Compliance with this type of diet is beneficial in reducing cardiovascular risk factors and also insulin requirement in some individuals

Fat: The recommended fat intake is $20 \%$ of the total energy intake. Individuals with diabetes have higher levels of plasma cholesterol and triglycerides and lower levels of high density lipoprotein than do nondiabetics. As a result, fat intake by diabetics should be modified both in quantity and quality.

Saturated and total fat should be restricted. The ratio of polyunsaturated to saturated $(P: S)$ of $2: 1$ is advisable. It is highly encouraged that fat intake should come from vegetables.
Monounsaturated fats should be used where possible. Use of processed foods containing saturated fats and trans-fatty acids should be limited. Eating fish-rich in omega-3 fatty acids at least once weekly is recommended.

Minerals and Vitamins: A diabetic under good metabolic control and adequate dietary intake needs no supplement. However, if a deficiency is demonstrated or when a person follows a very low energy diet in uncontrolled diabetes or those potential groups who are at risk, supplements may be needed

Fiber: A total dietary fiber intake of 30$40 \mathrm{~g}$ per day or $15-20 \mathrm{~g}$ for every 1000 calories is recommended. For children, $5 \mathrm{~g}$ plus $1 \mathrm{~g} /$ year age is suggested. Both soluble and insoluble fibers have been associated with improved outcome in people with diabetes.

Soluble fibers such as pectin and guar found in legumes, fruits and vegetables have been associated with reduction in serum cholesterol and improved blood sugar control. Insoluble fibres from cereals may reduce the risk for coronary heart disease and type 2 diabetes.

Starches and sugars are slowly absorbed when they are eaten as part of a high fibre diet. Including more foods and food combinations that combines cereal fibre with low glycemic index foods, may be helpful in optimizing health outcomes for people with diabetes or at risk of diabetes.

Alcohol: If it must be taken, it must always be ingested with a meal. Alcohol consumption should be limited to $5 \%$ of total energy intake or 1 to 2 glasses per day, whichever is less. It is contraindicated in conditions such as hypertriglyceridemia, obesity, neuropathy, poor glycemic control and 
pregnancy. If possible it should be avoided.

Sodium: People with diabetes are frequently hypertensive. Modest restriction of sodium intake can be beneficial to most diabetics. On the other hand, severe sodium restriction may be harmful in poorly controlled diabetes when there is postural hypertension and fluid imbalance. Daily intake of sodium is restricted to $1000 \mathrm{mg} / 1000 \mathrm{kcal}$ and not to exceed $3000 \mathrm{mg} /$ day.

Sweeteners: Their use is acceptable. However, their use must be in moderation to avoid any potential risk. Limited amounts of sucrose and other nutritive sweeteners may be used as part of an appropriate energy controlled, high fiber and low fat diet.

Pregnancy: Nutrition recommendation for women with pre-existing and gestational diabetes mellitus should be based on nutrition assessment. Monitoring blood glucose levels, urine ketones, appetite and weight gain can be a guide to developing and evaluating an appropriate individualized meal plan and to making adjustments to the meal plan throughout pregnancy to ensure desired outcomes.

Children: The diabetic child's diet follows the same general pattern as that for the adult. However, a child's energy and protein requirements per kilogram of body weight are higher to allow for proper growth and development.

Considerable conflicts arise regarding current dietary approach in young children with diabetes. It has been suggested that strict dietary disciplines inflict lasting psychological change on the child. Therefore some flexibility is required and as the child grows older, he/she should be encouraged. Special arrangement should be made for small lunches to be taken with readily assessed exchanges such as fruits or biscuits. Teenage revolt may include rejection of dietary disciplines with resultant diabetic influence but man y diabetic patients grow through this phase avoiding such crisis.

\section{CURRENT CONCEPTS: TOWARDS} A HIGH CARBOHYDRATE/HIGH FIBER/LOW FAT DIET

Current concept places emphasis on the glycemic response of various carbohydrate foods rather than the traditional belief about simple versus complex carbohydrate in the treatment of diabetes mellitus. There is now considerable evidence that different carbohydrate containing foods even though they contain similar amounts of carbohydrates by chemical analysis cause widely different blood glucose response after ingestion. The question is what is the glycemic index of food? It is the blood glucose of a food when compared to the level found after the ingestion of an equivalent amount of glucose. It is expressed in percentage.

Many factors together including carbohydrate type, type and amount of fiber, protein, fat, food form, method of preparation and role of enzyme inhibitors influence the glycemic index of a particular food.

Recent studies have shown that high carbohydrate/high fiber diets are effective in lowering blood glucose and are beneficial for the management of diabetes, especially in those with type 2 diabetes mellitus. The same foods that have low glycemic index also help to reduce blood cholesterol and triglyceride levels. Such foods should make up the major portion of the carbohydrate in the diet for diabetics. Long term studies show that that the glycemic index is a useful concept and 
the use of sucrose in moderate amount does not compromise glucose control. Therefore, it is time to forget the old distinction between starchy foods and sugary foods or simple versus complex carbohydrates. Hence the therapeutic division of carbohydrate by physiological response should be low glycemic versus high glycemic index carbohydrate.

Low fat diet: Numerous studies indicate that high fat diet can impair glucose tolerance and promote obesity, dyslipidemia and atherosclerotic heart disease. Research also shows these metabolic abnormalities are reversed or improved by reducing saturated fat intake. It has also been shown that improved control of cholesterol and lipids (for example, HDL, LDL and triglycerides) can reduce cardiovascular complications by 20 to $50 \%$. Choosing a lower fat, low cholesterol diet combined with regular exercise makes fat control more effective.

In view of the role of dietary fats on serum lipids concentration in the incidence of coronary heart disease, emphasis is now placed on diets that contain ratio of polyunsaturated to saturated fat $(P: S)$ of 2:1. Monounsaturated fat (such as canola, olive and peanuts oils) may have beneficial effects on triglyceride and glycemic control in some individuals with diabetes but care must be taken to avoid weight gain.

Polyunsaturated fats of omega-3 series provided naturally bin fish and other sea foods are beneficial in reducing serum glyceride without impairing glycemic control.

\section{CONCLUSION}

Nutrition management is a key component of long term health and quality of life for people with diabetes. It is important to note that the general principles for nutrition recommendations for people affected by diabetes are the same as those for the general population. The recommended diet can only be defined as a dietary prescription based on nutrition assessment and treatment goals.

Choosing a healthy diet with the right mixture of low and high glycemic index foods (and not just the traditional belief of emphasizing on simple versus complex carbohydrates in the treatment of diabetes), and exercising regularly is a good way to maintain glucose control.

Medical nutrition therapy for people with diabetes should be individualized with consideration given to usual eating habits and other lifestyle factors. Monitoring metabolic parameter including blood glucose, lipids, blood pressure, and body weight, as well as quality of life is crucial to ensure successful outcomes. It is important that the health care team work with people affected by diabetes to set realistic goals that meet the individual's micro and macronutrients, physical activity, lifestyle and medical needs. The registered dietitian is a key member of team supporting the individual in the attainment of these goals

\section{REFERENCES}

1. American Diabetes Associations Nutrition Recommendation and Principles for people with diabetes mellitus. Clinical Practice Recommendations 1997.

2. Astewart Truswell: ABC of Nutrition pp 84-86. 
3. Henrietta Nkechi Ene-Obong: Eating right (A Nutrition Guide) pp 130-133.

4. Krause and Mahan: Food, nutrition and diet therapy $\mathrm{pp}$ 487-491.
5. Umeh MJ. Dietary management of diabetes mellitus: a paper presentation, 2001.

6. Wolever $\mathrm{T}$ et al: Guidelines for the nutritional management of diabetes mellitus in the new millennium, Canadian Journal of Diabetes Care 1999. 